F. Gross, C. Osgood, and C.-C. Yang

Nagoya Math. J.

Vol. 47 (1972), 91-99

\title{
PRIME ENTIRE FUNCTIONS WITH PRESCRIBED NEVANLINNA DEFICIENCY
}

\author{
FRED GROSS, ${ }^{1}$ CHARLES OSGOOD, ${ }^{2}$ AND CHUNG-CHUN YANG ${ }^{2}$
}

\section{Introduction.}

According to [4] a meromorphic function $h(z)=f(g)(z)$ is said to have $f(z)$ and $g(z)$ as left and right factors respectively, provided that $f(z)$ is non-linear and meromorphic and $g(z)$ is non-linear and entire $(g$ may be meromorphic when $f(z)$ is rational). $h(z)$ is said to be $E$-prime ( $E$-pseudo prime) if every factorization of the above form into entire factors implies that one of the functions $f$, or $g$ is linear (polynomial). $h(z)$ is said to be prime (pseudo-prime) if every factorization of the above form, where the factors may be meromorphic, implies that one of $f$ or $g$ is linear (a polynomial or $f$ is rational).

Recently the following result was proved by Goldstein [3].

THEOREM 1. Let $F(z)$ be an entire function of finite order such that $\delta(a, F)=1$ for some $a \neq \infty$, where $\delta(a, F)$ denotes the Nevanlinna deficiency. Then $F(z)$ is E-pseudo prime.

The above theorem might suggest that for an entire function of finite order the existence of Nevanlinna deficiency and the primeness of a function are closely related to each other. The purpose of this note is to show that it is not the case in general. More precisely, we shall show the following:

THEOREM 2. Given any integer $k>0$, and constant $c, 0 \leq c \leq 1$, one can construct a prime function $f$ of order $k$ with $\delta(0, f)=c$.

Remark. By a well-known result of Nevanlinna [7] one sees immediately why the above result cannot hold for an arbitrary real positive $k$.

Received September 6, 1971.

1 Mathematics Research Center, Naval Research Laboratory, Washington, D.C., and University of Maryland, Baltimore County.

2 Mathematics Research Center, Naval Research Laboratory, Washington, D.C. 
The proof of Theorem 2 also yields the following result.

THEOREM 3. Given any $0 \leq c \leq 1$, there exist real constants $\lambda_{1}$ and $\lambda_{2}$ such that the function $F=z e^{\lambda_{1} e^{z}}\left(e^{\lambda_{2} e^{z}}+1\right)$ satisfies $\delta(0, F)=c$.

Theorem 3 gives us an example of functions of infinite order which are not pseudo-prime and which have a prescribed deficiency. The analogous problem for functions of finite order remains open.

\section{Definitions and preliminary lemmas.}

We shall say that a polynomial in $z$ with complex coefficients has property $R$ if (i) $p(z)$ is monic, (ii) $p(0)=0$, and (iii) for some sequence of points $\left(a_{r}\right)$ tending to $\infty$ each root of $p(z)-a_{r}=0$ lies on one of a finite number of fixed rays $r_{1}, \cdots, r_{l}$ out from $z=0$, for some positive integer $l$. If $z \in C, z \neq 0$, and $z=|z| e^{i \theta}$ where $-\pi \leq \theta<\pi$ we define $\arg (z)$ to be $\theta$.

LEMMA I. (i) The polynominal $p(z)$ has property $R$ if and only if $p(z)=z^{\frac{1}{2} k}\left(z^{\frac{1}{2} k}+b\right)$ for some $b \in C$ and positive integer $k$. (ii) If $b \neq 0$ all but at most a finite number of the $a_{r}$ lie on the ray defined by $\arg (z) \equiv 2(\arg (b))$ modulo $2 \pi$, while if $b=0$ the $a_{r}$ 's lie on any finite collection of rays out from $z=0$.

Proof. We shall first show the "if" part of (i). If $b=0$ this is trivial. If $b \neq 0$ set $b=|b| \varepsilon$. Choose the $a_{r}$ to all be of the form $a_{r}$ $=\left|a_{r}\right| \varepsilon^{2}$. Then we may write our equations as $\left(z^{\frac{1}{2} k} \varepsilon^{-1}\right)^{2}+|b|\left(z^{\frac{1}{2} k} \varepsilon^{-1}\right)=\left|a_{r}\right|$. Since $|b|^{2}+4\left|a_{r}\right|>0$ each $z$ which is a root must be such that $z^{\frac{1}{2} k} \varepsilon^{-1}$ is real. Thus the roots must lie on a finite number of rays out from $z=0$.

The greater part of this proof will be spent establishing the "only if" part of (i). In doing so we shall show, also, that if $p(z)$ has property $R$ then there exists a subsequence of the $a_{r}$ consisting only of points $a_{r}$ with each $\arg \left(a_{r}\right)=\alpha$ for some $-\pi \leq \alpha<\pi$. We shall now use this last assertion to help prove (ii) and shall then return to the proof of (i). If $b=0$ in (ii) there is nothing to prove. If $b \neq 0$ pass to a subsequence of the $\left(a_{r}\right)$ where each $\arg \left(a_{r}\right) \not \equiv 2(\arg (b))$ modulo $2 \pi$. (If this is not possible we are through.) We shall now obtain a contradiction. Note that as $\left|a_{r}\right|$ goes to $\infty$ the absolute values of the roots of $p(z)-a_{r}=0$ go to $\infty$ also. Now $\arg \left(a_{r}\right)=\arg \left(p\left(z_{1, r}\right)\right)$ where $p\left(z_{1, r}\right)=a_{r}$ and each $z_{1, r}$ belongs to the ray $r_{1}$, say. Thus 


$$
\begin{aligned}
\arg \left(a_{r}\right) & =\arg \left(\left(z_{1, r}^{\frac{1}{2} k}\right)\left(z_{1, r}^{\frac{1}{1} k}+b\right)\right) \\
& \equiv\left(k\left(\arg \left(z_{1, r}\right)\right)+\arg \left(1+b z_{1, r}^{-\frac{1}{2} k}\right)\right) \text { modulo } 2 \pi .
\end{aligned}
$$

Since $\arg \left(a_{\gamma}\right)$ and $\arg \left(z_{1, \gamma}\right)$ are constants then so is $\arg \left(1+b z_{1, r}^{-\frac{1}{2} k}\right)$. As $\left|a_{r}\right|$ goes to infinity $\left|z_{1, \gamma}\right|$ goes to infinity and $\arg \left(1+b z_{1, r}^{-\frac{1}{2} k}\right)$ goes to zero. Thus each $\arg \left(1+b z_{1, r}^{-\frac{1}{2} k}\right)=0$ so every $b z_{1, r}^{-\frac{1}{2} k}$ is real and each $b^{2} z_{1, r}^{-k}$ is positive. Also, from (1) we have now that

$$
\arg \left(a_{\gamma}\right) \equiv k\left(\arg \left(z_{1, \gamma}\right)\right) \text { modulo } 2 \pi
$$

so, since $b^{2} z_{1, \gamma}^{-k}$ is positive,

$$
\arg \left(a_{r}\right) \equiv 2(\arg (b)) \text { modulo } 2 \pi \text {. }
$$

This contradiction proves (ii) subject to our (as yet) unproven assertion.

We next begin the proof of the "only if" part of (i). Let us look at the $k$ different algebraic functions

$$
z_{j}(a)=\rho^{j} a^{k-1}+b_{0}+b_{-1} \rho^{-j} a^{-k-1}+\cdots
$$

for $(1 \leq j \leq k)$ which are roots of $p(z)=a$, where $\rho=\exp \left(2 \pi i k^{-1}\right)$ and the expressions are valid for all sufficiently large $|a|$. Let us now pass to a subsequence of the $\left(a_{r}\right)$ such that each series for $z_{j}\left(a_{r}\right)$ converges and each $\arg \left(z_{j}\left(a_{r}\right)\right)$ is constant (recall that there are only a finite number of values possible). Define $-\pi \leq \varepsilon_{j}(\gamma)<\pi$ by

$$
\arg \left(z_{j}\left(a_{r}\right)\right) \equiv\left(k^{-1} \arg \left(a_{r}\right)+j k^{-1}(2 \pi)+\varepsilon_{j}(\gamma)\right) \text { modulo } 2 \pi,
$$

for each $1 \leq j \leq k$. Note that for each $1 \leq j_{1}, j_{2} \leq k$

$$
\begin{aligned}
\varepsilon_{j_{1}}(\gamma)-\varepsilon_{j_{2}}(\gamma) \equiv & \left(\arg \left(z_{j_{1}}\left(a_{\gamma}\right)\right)-\arg \left(z_{j_{2}}\left(a_{\gamma}\right)\right)\right. \\
& \left.-\left(j_{1}-j_{2}\right) k^{-1} 2 \pi\right) \text { modulo } 2 \pi,
\end{aligned}
$$

and the right hand side above is a constant. Also each $\lim _{r \rightarrow \infty} \varepsilon_{j}(\gamma)=0$ since $\rho^{j}\left(a_{r}\right)^{k-1}$ is the dominant term of the expansion for $z_{j}\left(a_{r}\right)$ about infinity. Thus each $\lim _{r \rightarrow \infty}\left(\varepsilon_{j_{1}}(\gamma)-\varepsilon_{j_{2}}(\gamma)\right)=0-0=0$, so every $\varepsilon_{j_{1}}(\gamma)-\varepsilon_{j_{2}}(\gamma)$ $\equiv 0$ modulo $2 \pi$.

We now require that each $\left|a_{r}\right|$ be sufficiently large to guarantee that every $\left|\varepsilon_{j}(\gamma)\right|<k^{-1} \pi / 2$. Then every $\varepsilon_{j_{1}}(\gamma)-\varepsilon_{j_{2}}(\gamma)=0$. Set $\varepsilon(\gamma)=\varepsilon_{1}(\gamma)=\cdots$ $=\varepsilon_{k}(\gamma)$. Since $\pm a_{r}=\prod_{j=1}^{k} z_{j}\left(a_{r}\right)$ we have

$$
\arg \left(a_{\gamma}\right) \equiv \arg \left(a_{\tau}\right)+(k-1) \pi+k \varepsilon(\gamma) \operatorname{modulo} \pi,
$$


so $k \varepsilon(\gamma) \equiv 0$ modulo $\pi$. Thus $\varepsilon(\gamma)=0$ for all sufficiently large $\gamma$. Then by (2) with each $\varepsilon_{j}(\gamma)=0$ we see that $\arg \left(a_{\gamma}\right)$ is a constant on our subsequence. (This proves the statement needed in the proof of (ii).) From now on we assume that $k>2$, since there is nothing to prove if $k=2$. Also we have from (2) that, for each $1 \leq j \leq k$,

$$
\arg \left(z_{j}\left(a_{r}\right)\right) \equiv\left(k^{-1}\left(\arg \left(a_{r}\right)\right)+j k^{-1}(2 \pi)\right) \text { modulo } 2 \pi \text {. }
$$

Equation (4) says that each $z_{j}\left(a_{r}\right)$ has an argument equal to the argument of the dominant term in its expansion about $a_{r}=\infty$. We shall next show by induction that for all non-negative integers $n, b_{-n}=0$ unless $k$ divides $2(n+1)$. Further if $b_{-n} \neq 0$, then, for sufficiently large $\gamma$, $\arg \left(b_{-n}\left(\rho^{j} a_{r}^{k-1}\right)^{-n}\right) \equiv \arg \left(\rho^{j} a_{r}^{k-1}\right)$ modulo $\pi$. (Actually, we are only interested in proving the first statement but the second statement is needed in order to make the induction go through.) Since $k>2$ we must show that $b_{0}$ $=0$. Suppose $b_{0} \neq 0$, then for sufficiently large $\gamma$ we see that $z_{j}\left(a_{\tau}\right)-$ $\rho^{j}\left(a_{r}\right)^{k^{-1}}$ does not vanish so

$$
\begin{aligned}
k^{-1}\left(\arg \left(a_{r}\right)+j(2 \pi)\right) & \equiv \lim _{r \rightarrow \infty}\left(\arg \left(z_{j}\left(a_{\gamma}\right)\right)-\rho^{j}\left(a_{r}\right)^{k-1}\right) \\
& \equiv \arg \left(b_{0}\right) \text { modulo } \pi,
\end{aligned}
$$

for each $0 \leq j \leq k-1$. Since $k>2$ this is impossible. Thus $b_{0}=0$. Now assume the induction assumption for all $0 \leq l \leq n-1$ and that $b_{-n} \neq 0$. If $\gamma$ is sufficiently large $z_{j}\left(a_{r}\right)-\sum_{l=0}^{n-1} b_{-l}\left(\rho^{j} a_{j}^{k-1}\right)^{-l} \neq 0$ so that we have

$$
\begin{aligned}
k^{-1}\left(\arg \left(a_{r}\right)+j(2 \pi)\right) & \equiv \arg \left(z_{j}\left(a_{r}\right)\right) \text { modulo } 2 \pi \\
& \equiv \arg \left(z_{j}\left(a_{r}\right)-\sum_{l=0}^{n-1} b_{-l}\left(\rho^{j} a_{r}^{k-1}\right)^{-l}\right) \text { modulo } \pi \\
& \equiv \arg \left(b_{-n}\left(\rho^{j} a_{r}^{k-1}\right)^{-n}\right) .
\end{aligned}
$$

This proves the second statement in our induction assumption. Also we see from (5) that

$$
k^{-1}\left(\left(\arg \left(a_{r}\right)\right)(n+1)+j(2 \pi)(n+1)\right) \equiv \arg \left(b_{-n}\right) \text { modulo } \pi .
$$

Setting $j=1,0$ and subtracting we see that $k^{-1} 2(n+1)(\pi) \equiv 0$ modulo $\pi$. Therefore $k$ divides $2(n+1)$ if $b_{-n} \neq 0$. This completes the proof by induction.

We know that $p(z)-a=\prod_{j=1}^{k}\left(z-z_{j}(a)\right)$ and that each 


$$
\begin{aligned}
z_{j}(a)=\rho^{j} a^{k-1} & +b_{-\left(\frac{1}{2} k-1\right)}\left(\rho^{j} a^{k-1}\right)^{-\left(\frac{1}{2} k-1\right)}+b_{-(k-1)}\left(\rho^{j} a^{k-1}\right)^{-(k-1)} \\
& +O\left(\left(a^{k-1}\right)^{-\left(\frac{3}{2} k-1\right)}\right),
\end{aligned}
$$

where the last term indicates an infinite number of terms of order $\left(a^{k-1}\right)^{-\left(\frac{3}{2} k-1\right)}$ and lower. Since the coefficients of $p(z)$ are independent of $a$, if we put in the different series for the $z_{j}(a)$ in $\prod_{j=1}^{k}\left(z-z_{j}(a)\right)$ and find the total coefficient of $a^{0} z^{l}=z^{l}$, for $0<l<k-1$, we will have the coefficient of $z^{l}$ in $p(z)$. Our statement which must be demonstrated is that this coefficient vanishes if above $l \neq \frac{1}{2} k$. We shall show that it is impossible to find a term in the product above which equals a coefficient times $a^{0} z^{l}$, if $0<l<k-1$ and $l \neq \frac{1}{2} k$. It is clearly impossible to obtain such a term if we choose any factor from $O\left(\left(a^{k-1}\right)^{-\left(\frac{3}{2} k-1\right)}\right)$. Also choosing a factor of $\left(\rho^{j} a^{k^{-1}}\right)^{-(k-1)}$, for any $1 \leq j \leq k$, forces us to choose $k-1$ factors of the form $\left(\rho^{j_{1}} a^{k-1}\right)$ and forces $l$ to be zero. Thus the problem reduces to showing that one cannot find two non-negative integers $h_{1}$ and $h_{2}$ such that $0<h_{1}+h_{2}<k$ and $\left.\left(a^{k-1}\right)^{h_{1}}\left(a^{k-1}\right)^{-h_{2}\left(\frac{1}{2} k-1\right)}\right)=a^{0}=1$ unless $h_{1}+$ $h_{2}=\frac{1}{2} k$. Since $k>2, h_{2}$ can equal only either 1 or 2 . If $h_{2}=1$, then $h_{1}=\frac{1}{2} k-1$, so $h_{1}+h_{2}=\frac{1}{2} k$. If $h_{2}=2$ then $h_{1}=k-2$ so $h_{1}+h_{2}=k$, contrary to our assumption. This proves Lemma $I$.

LEMMA II. If $\alpha, \beta, \gamma$ are complex constants with $\beta \gamma \neq 0$ and $n$ is $a$ positive integer then $y=\gamma z\left(e^{\alpha z^{n}}+e^{\beta z^{n}}\right)$ takes on all values.

Proof. Suppose the statement is false. Then, by a result of Borel [1], one will obtain a contradiction. We leave the details to the reader.

LEMMA III. The function $y=\gamma z\left(e^{\alpha z^{n}}+e^{\beta z^{n}}\right)$ cannot be written in the form $p(g)$ where $g$ is entire, $p$ is any nonzero, nonlinear polynomial, $n$ is a positive integer, $\beta \gamma \neq 0$, and $\alpha \beta^{-1}$ is real.

Proof. We shall assume that $y=p(g)$ where $y, g$, and $p=p(w)$ are as above. This will lead us to the conclusion that $y$ takes on at least one value infinitely often with multiplicity larger than one; however, this latter conclusion will subsequently be shown to be false. Since $p(w)$ is nonlinear, $p^{\prime}(w)=0$ has at least one solution, $w_{0}$. Thus when $g(z)=$ $w_{0}$ we have that $y(z)=p\left(w_{0}\right)$ and has multiplicity greater than one. If $p^{\prime}(w)=0$ has two or more solutions $g$ cannot omit both roots, hence $y$ must take on the value of $p\left(w_{0}\right)$ infinitely often with multiplicity greater than one, for some $w_{0}$ such that $p^{\prime}\left(w_{0}\right)=0$. If $w_{0}$ is the only root of 
$p^{\prime}(w)=0$ then $p(w)=p^{(k)}\left(w_{0}\right)(k !)^{-1}\left(w-w_{0}\right)^{k}+p\left(w_{0}\right)$ for some positive integer $k \geq 2$. Then either $g$ takes on the value $w_{0}$ infinitely often (so that $y$ takes on the value $p\left(w_{0}\right)$ infinitely often with multiplicity greater than one) or $g$ takes on the value $w_{0}$ only finitely often (so $y$ takes on the value $p\left(w_{0}\right)$ only finitely often, since $p(w)-p\left(w_{0}\right)$ has only one zero). By Lemma II $y$ does not omit any values, therefore $y$ does assume some value $a=p\left(w_{0}\right)$ infinitely often with multiplicity greater than one. We shall next show that this is impossible.

It is necessary first to dispose of the special cases when $\alpha=0$ or $\alpha=\beta$. Suppose $\alpha=0$. Then replacing $z$ by $\sqrt[n]{\beta} z$ and then $p(w)$ by $\left(\gamma(\sqrt[n]{\beta})^{-1}\right)^{-1} p(w)$ we may assume, without loss of generality, that $y=$ $z\left(e^{z^{n}}+1\right)$. (Similarly, if $\alpha \beta^{-1}=1$, we may assume that $y=z e^{z^{n}}$.) Notice that $a \neq 0$, since if $z \neq 0, z\left(e^{z^{n}}+1\right)=0$, and $n z^{n} e^{z^{n}}+\left(e^{z^{n}}+1\right)=0$ we would have that $e^{z^{n}}=0$. If $a \neq 0$ then, for all nonzero $z$, if $y(z)=a$ and $y^{\prime}(z)=0$ we have $0=\left(y^{\prime}(z)\right)(y(z))^{-1}=z^{-1}+n z^{n-1} e^{z^{n}}\left(e^{z^{n}}+1\right)^{-1}=z^{-1}+$ $n z^{n} e^{z^{n}} a^{-1}=z^{-1}+n z^{n-1}(a-z) a^{-1}=z^{-1}+n z^{n-1}-n a^{-1} z^{n}$. For fixed $a \neq 0$ this equation has at most $n+1$ distinct solutions. Suppose that $z e^{z^{n}}=$ $a, e^{z^{n}}+n z^{n} e^{z^{n}}=0$, and $z \neq 0$. Then $z^{-1} a+n z^{n-1} a=0$. Since $z \neq 0$ we see that $a \neq 0$. Thus we have $z^{-1}+n z^{n-1}=0$ which can have at most $n$ distinct solutions.

If $\alpha \beta \gamma \neq 0$ and $\alpha \beta^{-1} \neq 1$, then without loss of generality we may take $y$ to be of the form $y=z\left(e^{\lambda z^{n}}+e^{z^{n}}\right)$ where $\lambda<1$ but $\lambda \neq 0$. Suppose $a=0$. Then requiring that $z \neq 0$, the equations $z\left(e^{\lambda z^{n}}+e^{z^{n}}\right)=0$ and $\left(e^{\lambda z^{n}}+e^{z^{n}}\right)+n z^{n}\left(\lambda e^{\lambda z^{n}}+e^{z^{n}}\right)=0$ imply that $e^{\lambda z^{n}}=e^{z^{n}}=0$. This contradiction shows that $a \neq 0$. Now assuming that $a \neq 0$ and $z \neq 0$ we have $0=z^{-1}+n z^{n}\left(\lambda e^{\lambda z^{n}}+e^{z^{n}}\right)\left(z\left(e^{\lambda z^{n}}+e^{z^{n}}\right)\right)^{-1}=z^{-1}+n z^{n-1} a^{-1}\left(a+z(\lambda-1) e^{\lambda z^{n}}\right)$. Then $e^{\lambda z^{n}}=a\left(1+n z^{n}\right)\left(n z^{n+1}(1-\lambda)\right)^{-1}$, so substituting back in $z\left(e^{\lambda z^{n}}+e^{z^{n}}\right)$ $=a$ we have

$$
z\left(a\left(1+n z^{n}\right)\left(n z^{n+1}(1-\lambda)\right)^{-1}\right)+z\left(a\left(1+n z^{n}\right)\left(n z^{n+1}(1-\lambda)\right)^{-1}\right)^{\lambda-1}=a,
$$

for an appropriate choice of the $\lambda$-th root above. Regardless of this choice, however, we see upon taking absolute values that $\infty>|a| \geq$ $|z| \cdot\left|a\left(1+n z^{n}\right)\left(n z^{n+1}(1-\lambda)\right)^{-1}\right|^{\lambda-1}-|z| \cdot\left|a\left(1+n z^{n}\right)\left(n z^{n+1}(1-\lambda)\right)^{-1}\right| . \quad$ As $|z|$ goes to infinity the first term on the right hand side above goes to $+\infty$ while the second term remains bounded. This contradiction proves Lemma III.

The following lemma is essentially an observation out of Goldstein's proof of Theorem 1. 
LEMMA IV. Let $F(z)=z e^{z^{k}}\left(e^{a z^{k}}+1\right)$, where $k$ is a positive integer and $a$ is a positive real number. Then $F$ is $E$-pseudo prime.

Sketch of the proof. Set

$$
K(z)=\left(e^{a z^{k}}+1\right) .
$$

Then $\delta(-1, K)=1$, and so by virtue of a result of Edrei and Fuchs [2, pp. 281-283] the estimate [2, p. 281] holds for $K$ along a sequence of arcs and segments. Now we note along those arcs and segments $e^{z^{k}}$ is bounded. Hence the mentioned estimate holds not only for $K$ but also for $F(z)$. Then following Goldstein's argument we will arrive at the conclusion.

\section{Proof of Theorem2.}

First of all, it is easy to verify that for any non-zero constants $\lambda_{1}$ and $\lambda_{2}$ and any positive integer $k, F(z)=z e^{\lambda_{1} z^{k}}\left(e^{\lambda_{2} z^{k}}+1\right)$ cannot be periodic. Thus by virtue of a result of the first author [5], we need only to show that $F$ is $E$-prime.

When $c=0$ or $c=1$ we choose $F=z\left(e^{z^{k}}+1\right)$ or $F=z e^{z^{k}}$, respectively, and it is easy to verify that they are all prime functions of order $k$. Therefore, we restrict ourselves to the case $0<c<1$.

Let us choose

$$
F(z)=z e^{\lambda_{1} z^{k}}\left(e^{\lambda_{2} z^{k}}+1\right)
$$

where $\lambda_{1}>0$ and $\lambda_{2}>0$ are chosen such that $\frac{\lambda_{1}}{\lambda_{1}+\lambda_{2}}=c$. We claim that $f(z)$ is $E$-prime with $\delta(0, F)=c$. We first show that $F$ is $E$-prime. $F$ is $E$-pseudo prime by virtue of Lemma IV. By Lemma III, $F$ also cannot assume the form $F=p(g)$ with $p$ a polynomial and $g$ transcendental entire. Thus we only need to consider the possibility that $F$ can be factorized as

$$
F(z)=g(p(z))
$$

where $g$ is transcendental, and $p$ is a nonlinear polynomial. We may assume without loss of generality that $p(0)=0$ and that the leading coefficient of $p$ is one.

Now, according to Lemma 1 ,

$$
p(z)=z^{n / 2}\left(z^{n / 2}+b\right) .
$$


where $n$ is an integer and $b$ a constant. We claim that $n=1$. Suppose that $n \geq 2$. Then from (7) and (8) we have

$$
F(z)=z e^{\lambda_{2} z^{k}}\left(e^{\lambda_{2} z^{k}}+1\right) \equiv g\left(z^{n / 2}\left(z^{n / 2}+b\right)\right) .
$$

Now if $b \neq 0$, then $n$ has to be even. Let us substitute $z$ by $\zeta z$ into identity (9) where $\zeta$ is a ( $n / 2)$-th root of unity other than one when $n>2$, and substitute $z$ by $-z-6$ when $n=2$. Then by Borel's result mentioned earlier one will obtain a contradiction. If $b=0$, then $n$ can be even or odd. We again substitute $z$ by $\zeta z$ into identity (9) and obtain a contradiction unless $n=1$ which means $p(z)$ is linear. Thus we have also excluded the possibility (7). Hence $F$ is $E$-prime, therefore is also prime.

Now we proceed to show that $\delta(0, F)=c$. Let us choose a nonnegative number $\lambda$ such that $\lambda+\lambda_{1}=n \lambda_{2}, n$ a positive integer.

Multiplying $F$ by $e^{2 z^{k}}$ we have

$$
H(z)=e^{\lambda z^{k}} F=z e^{n \lambda_{2} z^{k}}\left(e^{\lambda_{2} z^{k}}+1\right),
$$

or

$$
H(z)=z f^{n}(z)(f(z)+1),
$$

where $f(z)=e^{\lambda_{2} z^{k}}$.

According to a result of Hayman [6, p. 7]

$$
\begin{aligned}
T(r, H)= & T\left(r, z f^{n}(z)(f(z)+1)\right) \sim T\left\{r, f^{n}(z)(f(z)+1)\right\} \\
& \sim(n+1) T(r, f) \sim \frac{(n+1) \lambda_{2}}{\pi} r^{k}, \quad \text { as } r \rightarrow \infty .
\end{aligned}
$$

Now we have by Nevanlinna's first fundamental theorem and equation (10) that

$$
\begin{aligned}
T(r, F)= & F\left(r, H e^{-\lambda z^{k}}\right) \\
& \geq T(r, H)-T\left(r, e^{-\lambda z^{k}}\right)+O(1) \\
& \geq \frac{(n+1) \lambda_{2}}{\pi} r^{k}-\frac{\lambda}{\pi} r^{k}+O(1) \\
= & \frac{\left(n \lambda_{2}+\lambda_{2}-\lambda\right)}{\pi} r^{k}+O(1) \\
& =\frac{\lambda_{1}+\lambda_{2}}{\pi} r^{k}+O(1) .
\end{aligned}
$$

On the other hand 


$$
\begin{aligned}
T(r, F)= & T\left(r, z e^{\lambda_{1} z^{k}}\left(e^{\lambda_{2} z^{k}}+1\right)\right) \\
& \leq T\left(r, e^{\lambda_{1} z^{k}}\right)+T\left(r, e^{\lambda_{2} z^{k}}\right)+O(\log r) \\
& \sim \frac{\lambda_{1}}{\pi} r^{k}+\frac{\lambda_{2}}{\pi} r^{k}+O(\log r) \\
= & \frac{\lambda_{1}+\lambda_{2}}{\pi} r^{k}+O(\log r) .
\end{aligned}
$$

Thus from (13), (14), and noticing the fact that $F$ is transcendental, we conclude

$$
T(r, F) \sim(1+o(1)) \frac{\left(\lambda_{1}+\lambda_{2}\right)}{\pi} r^{k} \quad \text { as } r \rightarrow \infty .
$$

Now the counting function $N\left(r, \frac{1}{F}\right)$ is equal to $N\left(r, \frac{1}{e^{\lambda_{2} z^{k}}+1}\right)$ which is asymptotic to $T\left(r, e^{\lambda_{2} z^{k}}\right)$ by Nevanlinna's second fundamental theorem.

Thus from this and (15) we have

$$
\begin{aligned}
\delta(0, F) & =1-\varlimsup_{r \rightarrow \infty} \frac{N(r, 1 / F)}{T(r, F)} \\
& =1-\varlimsup_{r \rightarrow \infty} \frac{\left(\lambda_{2} / \pi\right) r^{k}}{\left(\left(\lambda_{1}+\lambda_{2}\right) / \pi\right) r^{k}}=\frac{\lambda_{1}}{\lambda_{1}+\lambda_{2}}=c .
\end{aligned}
$$

The theorem is thus proved.

\section{REFERENCES}

[1] E. Borel, Sur les zéros des fonctions entières, Acta Math., 20 (1897), p. 387.

[2] A. Edrei and W. H. J. Fuchs, Valeurs déficientes et valeurs asymptotiques des fonctions mèromorphes, Comm. Math. Helv., 33 (1959), pp. 258-295.

[ 3 ] R. Goldstein, On factorisation of certain entire functions, J. Lond. Math. Soc., (2), 2 (1970), pp. 221-224.

[4] F. Gross, On factorization of meromorphic functions, Trans. Amer. Math. Soc., Vol. 131, No. 1, 1968.

[ 5 ] — - Factorization of entire functions which are periodic mod $\mathrm{g}$, Indian Journal of Pure nad Applied Mathematics, Vol. 2, No. 3, 1971, p. 568.

[ 6 ] W. K. Hayman, Meromorphic functions, Oxford, 1964.

[ 7 ] R. Nevanlinna, Le théorème de Picard-Borel et la thèorie des fonctions mèromorphes, Paris, Gauthier-Villars, 1929, p. 51. 